\title{
HUMAN TEAR FLUID DIAGNOSTIC ASSESSMENT ENABLED BY MICROFLUIDIC PROTEIN ELECTROPHORESIS
}

\author{
Kelly Karns, Aleksandra Denisin and Amy E. Herr \\ Department of Bioengineering, University of California, Berkeley, CA 94720, USA
}

\begin{abstract}
Human tear film fluid offers tremendous potential as a noninvasive diagnostic fluid. However, the use of tear fluid in diagnostic medicine is limited owing to non-standardized collection methodologies and low volume availability. We present a first-reported microfluidic tear fluid biomarker assay which will serve as a foundation for both rapid biomarker validation and point-of-care ocular diagnostics. Furthermore, we detail important advances in tear fluid preparation for high fidelity protein collection.
\end{abstract}

\section{INTRODUCTION}

Human tear film fluid contains numerous proteins that are specific to the eye and others that are indicative of systemic protein levels [1]. Importantly, several eye-specific tear proteins have been shown to be putative biomarkers for diseases ranging from autoimmune diseases (e.g., Sjogren's syndrome) to ocular bacterial infections rampant in the developing world (e.g. trachoma) [2,3]. Furthermore, other proteins such as albumin exist in tear fluid at levels equal to those in serum, offering the potential to use tear fluid to monitor and track systemic protein expression levels. As a result, human tear fluid has significant potential as a non-invasive diagnostic fluid [1]. Despite the promise it offers, the use of tear fluid in diagnostic medicine is limited [4].

Major technological hurdles hinder routine clinical assessment of tear film fluid proteins. These gaps include: low throughput ( $>5$ hours for analysis), lack of automation potential, consumption of large sample volumes, and limited sensitivity levels for clinical assessment. Taken together, these gaps make current assays (i.e., sizing, ELISA) unsuitable for point-of-care diagnostics and rapid endogenous protein quantification in clinically relevant ranges. Furthermore, tear collection methodologies are poorly characterized and highly variable, yielding widely disparate starting sample compositions which confound research findings and limit biomarker-based diagnostic measurements. Consequently, efficient, reliable sample preparation and rapid, quantitative, highly specific assays for endogenous tear proteins would impact a spectrum of needs: from our basic understanding of ocular disease to the possibility of point-of-care diagnosis and treatment.

In particular, a rapid biomarker-based assay for the diagnosis of Sjogren's syndrome (SS) would provide tremendous clinical benefit. SS is a severe form of keratoconjunctivitis sicca (KCS or dry eye disorder) and is a systemic autoimmune disease that is estimated to impact $1-2 \%$ of the general population [5]. It causes severe dry eye and xerostomia (dry mouth arising from reduced saliva production) and is associated with vision threatening complications including scleritis (a serious inflammatory disease that affects the white outer coating of the eye), corneal ulceration and secondary bacterial infection [6]. Early diagnosis is important for timely disease management and the prevention of permanent tissue damage. However, current diagnostics are complicated by the absence of universal diagnostic criteria, the requirement of an invasive salivary gland biopsy and the similarity of symptoms with those of other common disorders. Consequently, average time to diagnosis after the onset of symptoms is over 6 years [7].

Lactoferrin (Lf) stands out as a high-abundance tearspecific protein which is down-regulated in the tears of patients with KCS and SS when compared with healthy controls and down-regulated in tears of patients with SS when compared with non-SS KCS patients [8]. Importantly, the development of a biomarker-based assay for the differential diagnosis of SS and severe KCS would have real clinical benefit by eliminating the need for salivary gland biopsy and promoting timely treatment of glandular and extraglandular manifestations of SS. It would also open up the possibility of presymptomatic diagnosis of autoimmune diseases such as rheumatoid arthritis, which occur in $60 \%$ of SS patients [6]. Owing to the clinical benefits of such a test as well as the identification of Lf as a possible biomarker for SS, Lf was chosen as a biomarker to validate the technology platform performance.
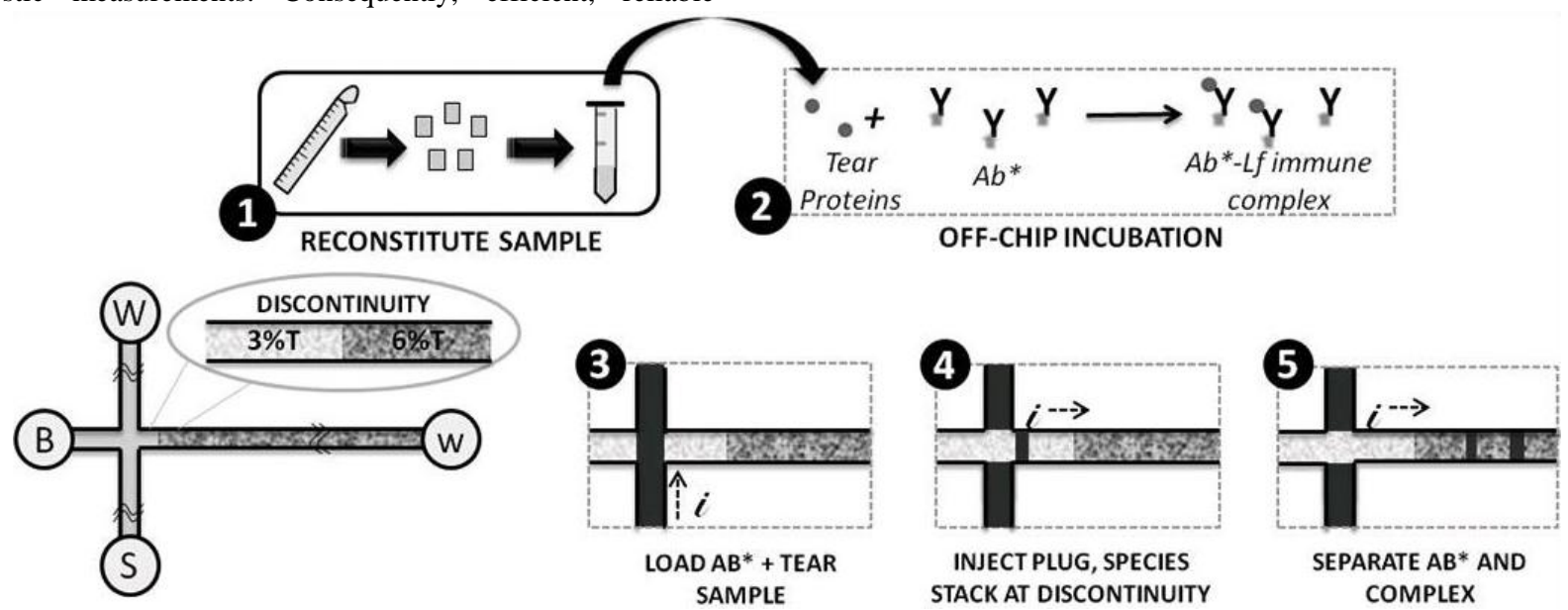

Figure 1: Microfluidic protein electrophoresis enables the rapid quantification of endogenous volume-limited tear fluid proteins. (1) Tear proteins are reconstituted from Schirmer strips. (2) Lf-containing tear fluid is incubated with labeled antibody (Ab*) and loaded on chip (well S). (3) Sample is electrophoretically loaded into loading channel. (4) Finite plug is injected into separation channel and stacked at discontinuity (5) $A b^{*}$ and complex peaks are resolved. A $b^{*}$-Lf complex formation detects presence of targeted proteins in tear sample. 
This paper presents important advances in tear fluid preparation for high fidelity protein collection and a first-in-kind rapid microfluidic tear fluid biomarker assay (Lf). Importantly, while the focus in the present study is measurement of Lf, other important protein markers of disease are amenable to measurement using the approach. To our knowledge, no similar efforts exist. The following sections present theory and device design followed by fabrication technique, experimental results and finally the relevant discussion and conclusions.

\section{THEORY AND DEVICE DESIGN}

Microfluidic electrophoretic immunoassays are uniquely suited to rapidly quantify endogenous proteins in volumelimited tear samples. The approach takes advantage of the high specificity of antibody-antigen interactions to separate fluorescently labeled free antibody from bound antibody-antigen immune complex for a quantitative measure of antigen concentration in a complex sample. These immunoassays offers numerous advantages over current techniques including rapid species resolution (seconds), high analytical specificity, low sample volume requirements (nL to $\mu \mathrm{L}$ ), the potential for facile multiplexing to assess multiple proteins in a single sample, and a small form factor for point-of-care, automated use.

For use as a differential diagnostic for SS and KCS, the assay was optimized to ensure detection of clinically relevant Lf concentrations. Previous reports have shown the mean concentration of Lf in SS tears to be $1 \mu \mathrm{M}$ Lf, while non-SS KCS tears have a mean Lf concentration of $8 \mu \mathrm{M}$ [8]. Allowing for a $10 \mathrm{x}$ dilution factor in sample preparation, this suggests $100 \mathrm{nM}$ Lf as a starting diagnostic threshold on which to center the detectable assay range.

A polyacrylamide gel was used within the channels as shown previously [9] and the gel pore size was tuned in order to enhance sensitivity. Photopatterned discontinuous gel architectures in particular have recently been shown by our group to enhance sensitivity levels and allow for ultra short separation distances, opening the possibility for point-of-care, battery powered devices that employ analytical grade electrophoresis [10]. Sample stacking occurs at the pore discontinuity and is proportional to the extent of pore size change as given in Equation 1

$$
\log \left(\frac{C_{1}}{C_{2}}\right)=-k_{r}\left(T_{2}-T_{1}\right)
$$

where $C_{1}$ and $C_{2}$ are the average plug concentrations before and after the discontinuity respectively, $k_{\mathrm{r}}$ is the retardation coefficient of the analyte and $T_{1}$ and $T_{2}$ are the total acrylamide concentrations before and after the discontinuity respectively. The change in total acrylamide concentration was therefore maximized to achieve the greatest possible sample stacking and enhanced sensitivity. A discontinuous gel architecture of 3-6\% T was experimentally determined to allow for maximal sample stacking and resolution while still allowing for the migration of both free antibody and complex.

Figure 1 presents a schematic overview of the final assay concept. Key design features include: (i) large pore-size loading gel for non-biased sample loading, (ii) pore-size discontinuity for sample stacking and enhanced resolution and sensitivity and (iii) smaller pore-size separation gel for rapid species resolution.

\section{FABRICATION}

A 3-6\% $\mathrm{T}$ discontinuous polyacrylamide gel architecture was fabricated and optimized to separate fluorescently-labeled anti-human Lf antibody $\left(\mathrm{Ab}^{*}\right)$ from the Lf immune complex. Figure 2 shows the two step photopolymerization protocol as described previously [10]. Briefly, 6\% T precursor solution was loaded onto a standard T-channel networked glass microfluidic chip. A small region immediately after the injection junction was polymerized using a photomask and UV objective for $3 \mathrm{~min}$ at $5.8 \mathrm{~mW} / \mathrm{cm}^{2}$. The precursor solution in the loading channels were then replaced with a $3 \% \mathrm{~T}$ precursor solution and the chip was flood exposed on a UV lamp for $7 \mathrm{~min}$ at $12 \mathrm{~mW} / \mathrm{cm}^{2}$. The result was a sharp discontinuity in pore size $600 \mu \mathrm{m}$ after the injection junction.

\section{RESULTS AND DISCUSSION \\ Microfluidic Immunoassay}

Using this new approach, we have demonstrated the ability to detect and quantify endogenous $\mathrm{Lf}$ in $\langle 1 \mu \mathrm{L}$ of healthy and SS tears using a 3-6\% T discontinuous gel architecture. Figure 3 shows time-based montages of the electrophoretic separation of $\mathrm{Ab}^{*}$ in dilute healthy tears and dilute SS tears compared to buffer. In both the healthy and SS tear samples, the unbound antibody is clearly separated from the $\mathrm{Ab}^{*}$-Lf immune complexes which are retarded at the discontinuity, indicating the detection of endogenous Lf in healthy and SS tears. In Figure $4 \mathrm{~A}$, fluorescence intensity along the channel is plotted at $19 \mathrm{~s}$ for three samples: $\mathrm{Ab}^{*}$ in buffer, $\mathrm{Ab}^{*}$ in healthy tears, and $\mathrm{Ab}^{*}$ in

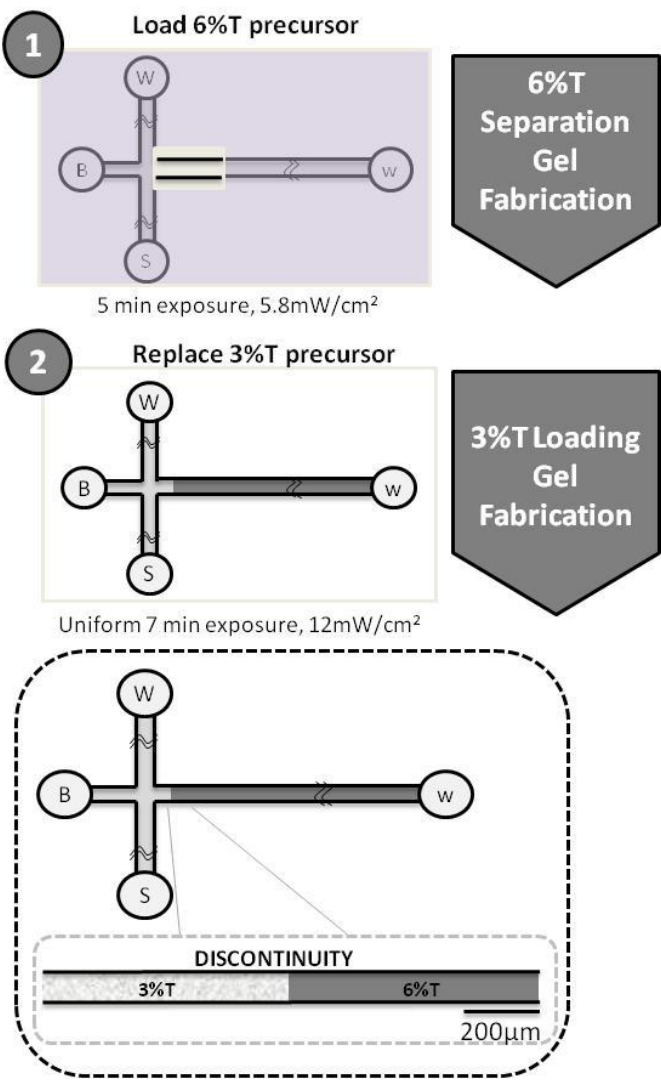

Figure 2: Two step photopatterning polymerization fabrication protocol yields sharp pore size discontinuity that enhances assay sensitivity and resolution efficiency. $6 \% T$ precursor is loaded into chip and small region is exposed on a UV objective for $3 \mathrm{~min}$ at $5.8 \mathrm{~mW} / \mathrm{cm}^{2}$. Solution in loading channels was then replaced with a $3 \% T$ precursor and the whole chip flood exposed for $7 \mathrm{~min}$ at $12 \mathrm{~mW} / \mathrm{cm}^{2}$. 
SS tears. When $\mathrm{Ab}^{*}$ binds to endogenous Lf in tear fluid, there is a decrease in free antibody in solution and an immune complex peak appears, yielding a quantitative measure of Lf. Importantly, we note the following: (i) a complex peak emerges in both SS and healthy tears when compared to $\mathrm{Ab}^{*}$ in buffer, indicating the detection of endogenous Lf in both SS and healthy tears, (ii) the complex peak in SS tears is lower than that of healthy tears, demonstrating a decrease in endogenous Lf in SS tears compared to healthy tears and validating the clinical observation that Lf is down-regulated in SS tears, and (iii) unlike in conventional slab gel measurements (data not shown), multiple complex bands can be resolved in the assay, providing additional information about tear composition and binding configurations. Gold-standard ELISA measurements confirm the data observed in the immunoassay assessment. Critical for point-of-care diagnostics, the Lf immunoassay completes in $<20$ s.
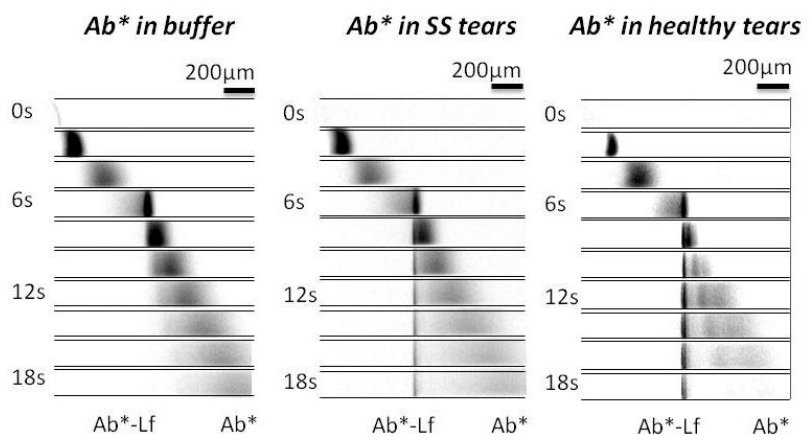

Figure 3: Endogenous Lf is detected in healthy and diseased tears in $<20 \mathrm{~s}$ with a 700 $\mu \mathrm{m}$ separation distance. $A b^{*}$ is incubated in buffer, dilute SS tears and dilute healthy tears and electrophoretically separated. Time-evolving fluorescence images are shown for the first $18 \mathrm{~s}$. A $b^{*}$ and $A b^{*}-L f$ complexes are resolved in $700 \mu \mathrm{m}$.

To provide an accurate quantitative measure of $\mathrm{Lf}$ in solution, increasing amounts of Lf were spiked into diluted SS tears and separated on-chip. Complex peak heights were extracted from the fluorescence images as shown in Figure 4B and a nonlinear least-squares fit with a 3-parameter model was used to establish the relationship between Lf concentration and complex peak height $(\beta 1=882.8, \beta 2=69.6, \beta 3=30.2)$. The model is predictive to within $12 \%$ in the clinically relevant range of 15-230nM Lf. Quantification of endogenous Lf in SS tears is confirmed to within $8 \%$ of gold-standard ELISA measurements.

Assay specificity is also crucial for a diagnostic measurement. Especially due to the complexity and relative unknown composition of tear fluid, efforts must be made to control for any non-specific interaction of anti-human $\mathrm{Lf} \mathrm{Ab}^{*}$ with other components of tears. Samples of $\mathrm{Ab}^{*}$ and a protein ladder containing known proteins trypsin inhibitor, parvalbumin and anti-human IgG antibody were separated independently on chip and then incubated together and separated simultaneously. A lack of additional peaks in the simultaneous separation gives a preliminary indication that nonspecific binding is not occurring between $\mathrm{Ab}^{*}$ and these proteins (Figure 5) while incubation of $\mathrm{Ab}^{*}$ with Lf has shown binding both in on-chip and bench-top studies.

Further work will include incubation of $\mathrm{Ab} *$ in Lf-depleted tear fluid to probe for nonspecific binding interactions.
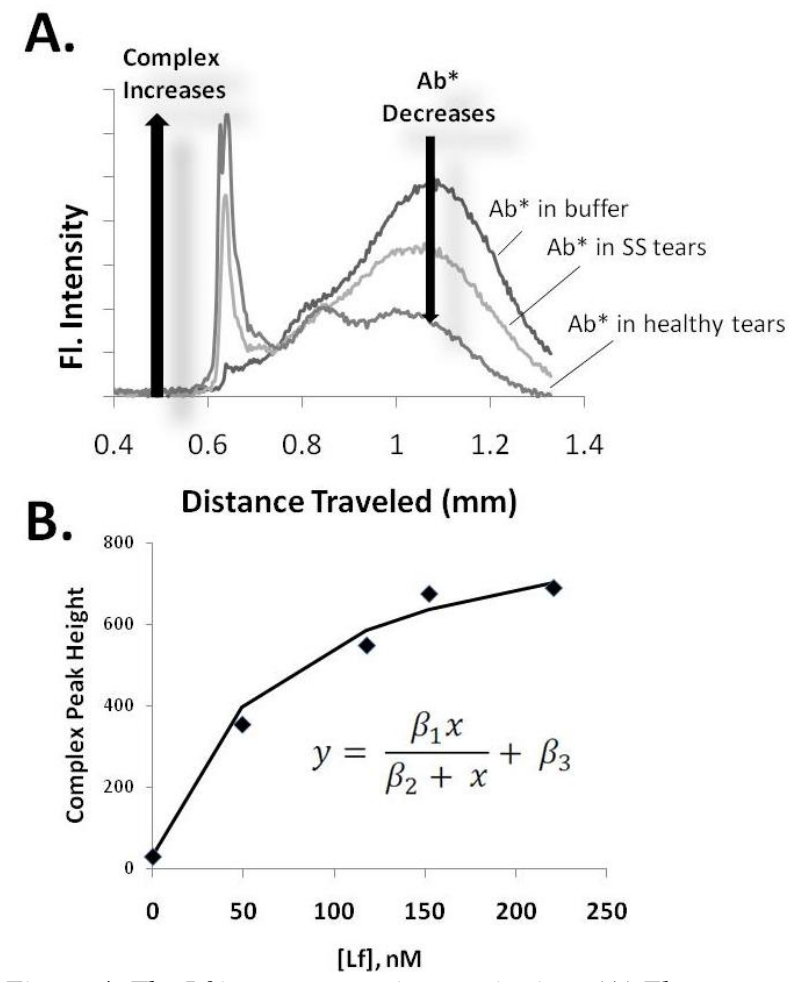

Figure 4: The Lf immunoassay is quantitative. (A) Fluorescence intensity in the channel is plotted at $19 \mathrm{~s}$ for three samples: A ${ }^{*}$ in buffer, $A b^{*}$ in healthy tears, and $A b^{*}$ in SS tears. A complex appears in tear fluid, yielding a quantitative measure of endogenous Lf in SS and healthy tears. (B) Lf immunoassay is quantitative for endogenous $L f$ in tear fluid to within $12 \%$. Lf dose response curve generated by on-chip immunoassay with 3parameter model fit given by equation.

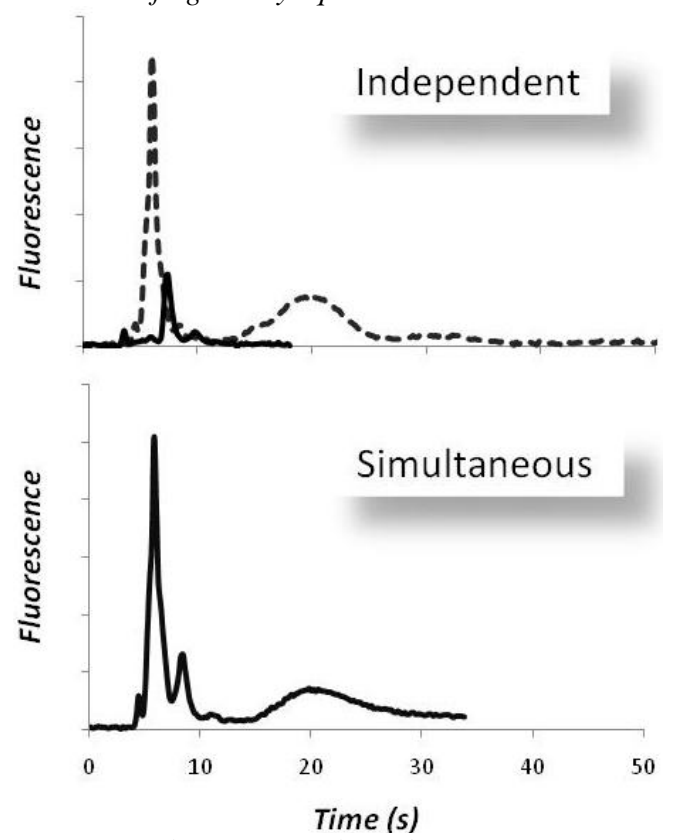

Figure 5: Preliminary results indicate assay specificity. Labeled protein ladder (dashed line) and $\mathrm{Ab} *$ (solid) are separated independently and then incubated and separated simultaneously. A lack of added peaks shows preliminary indication of assay specificity. 


\section{Sample Preparation}

For a biomarker-based immunoassay to yield meaningful clinical results it must utilize a standardized sample preparation methodology. Currently, novel tear-based diagnostic assays are complicated by the difficulties of collecting tear fluid in sufficient volumes and standardized composition. The clinical standard for tear collection and storage in large patient registries (e.g. UCSF) is the Schirmer strip; yet these strips are intended for volumetric measurements of tear production for diagnostic purposes and not for protein collection and extraction. Since assay reliability is only as good as upstream sample preparation, an efficient method of reconstituting tear proteins from Schirmer strips is paramount to a successful diagnostic assay. Key reconstitution metrics include: i) minimal sample dilution, ii) high efficiency extraction and iii) comparison of efficiencies across different proteins and concentrations.

To optimize and characterize the reconstitution protocol, we used BSA as a model protein to establish the reconstitution efficiency of protocols found in the literature $[11,12,13,4]$. Tested variables included altered buffer conditions, elution times and centrifugation vs. diffusion-based extraction. The optimized protocol involves incubating the strips in phosphate-buffered saline (PBS) for 3 hours at room temperature while shaking. Using this protocol, we have been able to demonstrate $78-100 \%$ extraction efficiencies across a concentration range of 0.75 $2 \mathrm{mg} / \mathrm{mL}$ with a $2 \mathrm{x}$ dilution factor during reconstitution (Figure 6). Results are on par with most efficient reported extraction protocols to date [14]. Future work in this area will include expanding this study to other proteins, complex samples and lower concentrations, closer to the protein levels found in tear fluid.

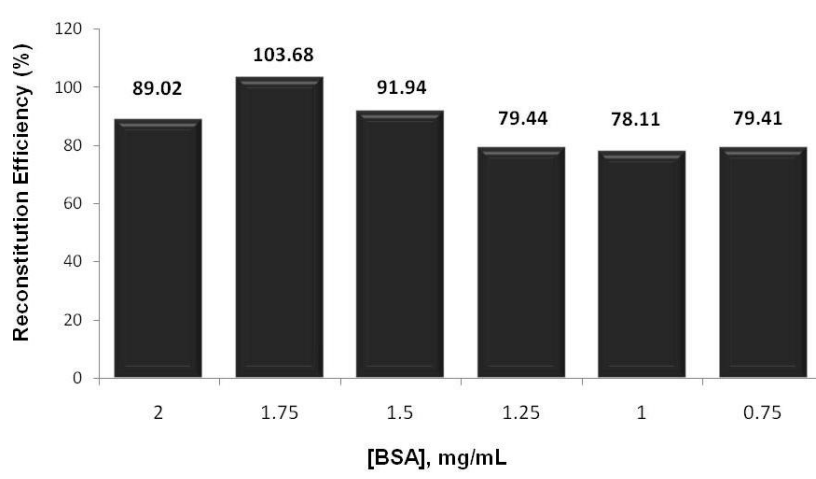

Figure 6: Optimized sample reconstitution protocol yields 78 $100 \%$ efficiencies with minimal $(2 x)$ dilution.

To our knowledge, this study represents the first use of tear fluid to diagnose systemic disease and opens up the possibility of an entirely new non-invasive diagnostic sample matrix. The technology platform can be expanded to other diseases by changing the biomarker or multiplexing. As such, we anticipate that it will have a large impact on the research and clinical community by advancing our basic understanding of ocular and systemic disease as well as opening the possibility of point-ofcare diagnosis and treatment.

\section{CONCLUSIONS}

For the first time, we demonstrate technologies critically needed for non-invasive tear-based diagnostics. Namely, we report on improved sample preparation for protein extraction and the first known endogenous microfluidic immunoassay for protein biomarker detection and quantitation in human tears.
Importantly, the microfluidic protein assay requires seconds to complete and microliter sample volumes, providing the foundation for first-in-kind, highly specific, rapid clinical diagnostics relevant to ocular and systemic diseases.

\section{ACKNOWLEDGEMENTS}

The authors thank Ms. Chenlu Hou (UC Berkeley) and Dr. Nancy McNamara (UCSF Proctor Eye Foundation) for technical assistance. Financial support provided by the DARPA Young Faculty Award (AEH) and an NSF GRF (KK).

\section{REFERENCES}

[1] Mann A.M, Tighe B.J., "Tear Analysis and Lens-Tear Interactions Pt.1: Protein Fingerprinting with Microfluidic Technology." Cont Lens Anterior Eye. 30, 163 (2007).

[2] Wu K., Zhang Y., "Clinical Application of Tear Proteomics: present and future prospects", Proteomics Clin. Appl. 1, 972 (2007).

[3] Dean D., Kandel R.P., Adhikari H.K., Hessel T., "Multiple Chlamydiaceae Species in Trachoma - Implications for Disease Pathogenesis and Control", PLOS Med. 5, 57 (2008).

[4] Zhou L., Beuerman R.W., Chan C.M., Zhao S.Z., Li X.R., Yang H., Tong L., Liu S., Stern M.E., Tan D., "Identification of Tear Fluid Biomarkers in Dry Eye Syndrome Using iTRAQ Quantitative Proteomics", J. Proteome Res. 8, 4889 (2009).

[5] Asbell P.A., Lemp M.A., Dry Eye Disease: The Clinician's Guide to Diagnosis and Treatment. Thieme Medical Publishers, NY (2006)

[6] Lemp M.A., "Epidemiology and Classification of Dry Eye", Technical Digest of the $2^{\text {nd }}$ International Conference on the Lacrimal Gland, Tear Film, and Dry Eye Syndromes, Southampton Princess Resort, Bermuda (1996) pp. 791-803

[7] Sjogren's Syndrome Foundation. "Diagnosis" http://www.sjogrens.org/home/about-sjogrenssyndrome/diagnosis

[8] Ohashi Y, Ishida R, Kojima T, Goto E, Matsumoto Y, Watanabe K, Ishida N, Nakata K, Takeuchi T, Tsubota K, Abnormal Protein Profiles in Tears with Dry Eye Syndrome", AJO, 136, 291 (2003)

[9] Herr A.E., Sing, A.K. "Photopolymerized Cross-Linked Polyacrylamide Gels for On-Chip Protein Sizing”, Anal Chem, 76, 4727 (2004)

[10] Hou. C., Herr A.E. "Ultrashort Separation Length Homogeneous Electrophoretic Immunoassays Using OnChip Discontinuous Polyacrylamide Gels", Anal Chem, accepted (2010)

[11] Li K, Chen Z, Duan F, Liang K.W. "Quantification of tear proteins by SDS-PAGE with an internal standard protein: A new method with special reference to small volume tears", Graefes Arch Clin Exp Ophthalmol, 10, 107 (2009)

[12] Mackie I.A., Seal D.V. "Diagnostic Implications of Tear Protein Profiles", British Journal of Ophthalmology, 68, 321 (1984)

[13] Remington S.G., Crow J.M., Nelson J.D., "Autoantibodies to Endogenous Tear Protein in Normal Human Tears", Current Eye Research, 34, 819 (2009)

[14] A. Kijlstra, S.H.M. Jeurissen, K.M. Koning, "Lactoferrin Levels in Normal Human Tears”, BJO. 67, 199 (1983). 